\title{
Philosophiques
}

\section{Louise Poissant, Machinations, Montréal, Société d'Esthétique du Québec, 1989, 128 p.}

\section{Jacques G. Ruelland}

Volume 17, numéro 1, printemps 1990

URI : https://id.erudit.org/iderudit/027114ar

DOI : https://doi.org/10.7202/027114ar

Aller au sommaire du numéro

Éditeur(s)

Société de philosophie du Québec

ISSN

0316-2923 (imprimé)

1492-1391 (numérique)

Découvrir la revue

Citer ce compte rendu

Ruelland, J. G. (1990). Compte rendu de [Louise Poissant, Machinations,

Montréal, Société d'Esthétique du Québec, 1989, 128 p.] Philosophiques, 17(1),

161-162. https://doi.org/10.7202/027114ar d'utilisation que vous pouvez consulter en ligne.

https://apropos.erudit.org/fr/usagers/politique-dutilisation/ 
LOUISE POISSANT, Machinations, Montréal, Société d'Esthétique du Québec, $1989,128 \mathrm{p}$.

\author{
Jacques G. Ruelland
}

En 1984, la Société d'Esthétique du Québec avait publié un excellent catalogue intitulé L'Art pense, pour l'exposition du même nom où une pléiade d'artistes proposaient leur définition personnelle de l'art. La qualité des textes de ce catalogue rehaussait les illustrations et rendait encore plus vivante la dynamique artistique que l'ouvrage désirait entretenir par sa facture utilitaire.

Le même commentaire s'applique à Machinations, tout en introduisant le lecteur à une nouvelle dimension du support artistique: l'ordinateur. Macbinations rend compte en effet d'une exposition d'auvres encore «en quête d'identité » (p. 3).

Dans son introduction (pp. 3-9), Louise Poissant dépeint les multiples conditions d'apparition de l'ordinateur dans le domaine de la production artistique : ses exigences institutionnelles et esthétiques, l'abandon de l'autonomie et de la pureté formaliste, de la gestuelle et de l'expressivité (p. 3) ; les grands espoirs que suscite ce nouveau médium et les maigres résultats que son exploitation génère ; la nécessité où se trouve le peintre ou le sculpteur de renouveler sa palette ou son burin, et surtout la réticence du public devant cette forme d'art qui semble trop souvent se faire l'apologiste d'une idéologie techniciste plutôt que de témoigner d'une recherche artistique aussi pure que traditionnelle.

Machinations - tant le catalogue que l'exposition elle-même - démontre que l'artiste qui fait appel à l'ordinateur a abandonné son image romantique, a perdu son inspiration artistique d'autrefois, et a coupé les paramètres de l'esthétique (p. 5). L'essentiel de la démarche et de la recherche artistiques actuelles ne porte plus sur le produit, mais sur le processus de production luimême (p. 5). C'est pourquoi "ce qui est en jeu derrière la diversité des procédés utilisés, c'est la nature de la présence de l'art» (p. 7), et non simplement la place de l'œuvre d'art dans la société, débat déjà fort vieux et peut-être même dépassé. La société actuelle ne peut ignorer le reflet que lui renvoie le miroir de l'art: la transformation de la raison instrumentale, du cadre référentiel et des variables du jugement esthétique.

Herbert Read, dans La philosophie de l'art moderne (Paris, Sylvie Messinger, 1988, p. 82), déclare que « le critère de l'artiste moderne est la Vérité plutôt que la Beauté. Ainsi, l'art moderne va de pair avec les sciences modernes ». Cette déclaration vaut certainement pour toute la recherche picturale précédant l'apparition du cubisme; elle ne peut guère s'appliquer telle quelle à une forme d'art comme celle qu'expose Machinations. Si l'on remplace l'adjectif « moderne » de cette phrase par l'idée d'utiliser l'ordinateur, et l'expression «sciences modernes » par la recherche d'un médium susceptible de faciliter l'expression des nouvelles valeurs techno-scientifiques de notre 
époque, alors Read a raison. Mais son idée a complètement changé de sens : la Beauté n'est plus en la chose, elle est la condition préexistante à cette chose, elle réside dans la recherche et dans le processus de production de l'œuvre, et non plus dans le produit fini.

C'est cela que démontre ce livre, qui, malgré sa destination finale, semble être plus qu'un simple catalogue.

L'exposition Machinations fut montrée à la Galerie de l'Université du Québec à Montréal en janvier 1989. Les exposants: Tony Brown, Diana Burgoyne, Claude Courchesne, Gervais Deschênes, Michel Dubreuil, Laura Kikauka, Serge Lemoyne, Nancy Paterson, Laurent Pilon, David Rokeby, Tom Sherman, Nell Tenhaaf, Agnès Tremblay, Normand White.

Département de philosophie

Collège Édouard-Montpetit 